\title{
Quantification of strain analysis and late gadolinium enhancement in coronary chronic total occlusion: a cardiovascular magnetic resonance imaging follow-up study
}

\author{
Lijun Zhang', Jinfan Tian², Xueyao Yang ${ }^{2}$, Jielin $\mathrm{Liu}^{3}$, Yi He ${ }^{4}$, Xiantao Song ${ }^{2}$ \\ ${ }^{1}$ Department of Radiology, Beijing Anzhen Hospital, Capital Medical University, Beijing, China; ${ }^{2}$ Department of Cardiology, Beijing Anzhen \\ Hospital, Capital Medical University, Beijing, China; ${ }^{3}$ Center for Cardiopulmonary Research, Beijing Anzhen Hospital, Capital Medical University, \\ Beijing, China; ${ }^{4}$ Department of Radiology, Beijing Friendship Hospital, Capital Medical University, Beijing, China
}

Contributions: (I) Conception and design: Y He, X Song; (II) Administrative support: X Song; (III) Provision of study materials or patients: Y He, X Song; (IV) Collection and assembly of data: L Zhang, J Tian, X Yang; (V) Data analysis and interpretation: L Zhang, J Tian, J Liu, Y He; (VI) Manuscript writing: All authors; (VII) Final approval of manuscript: All authors.

Correspondence to: Yi He. Department of Radiology, Beijing Friendship Hospital, Capital Medical University, Beijing, China. Email: Heyi139@sina.com; Xiantao Song. Department of Cardiology, Beijing Anzhen Hospital, Capital Medical University, Anzhenli Avenue, Chao Yang District, Beijing 100029, China. Email: songxiantao0929@qq.com.

Background: The present study aimed to investigate the benefits of percutaneous coronary intervention (PCI) in patients with chronic total occlusions (CTOs) by using cardiac magnetic resonance imaging (CMR) feature tracking.

Methods: Fifty-five CTOs with successful CTO-PCI underwent CMR at baseline and 12 months. Feature tracking was applied to measure left ventricle strain index in CTOs with decreased and preserved left ventricular ejection fraction (LVEF). CTOs were also divided into two groups according to the infarct size of $10 \%$ or combined with multi-vessel disease. We also measured these parameters in 40 healthy subjects.

Results: Three quarters of CTOs showed preserved ejection fraction and no enlargement of left ventricle at baseline, but the global strains were lower than the controls (all $\mathrm{P}<0.01$ ). In the entire CTO population, left ventricular ejection fraction did not show significant improvement in the 1 -year follow-up $(59.8 \% \pm 11.3 \%$ vs. $62.0 \% \pm 8.6 \%, \mathrm{P}=0.08)$. However, global strains improved over time, and peak global radial strain and circumferential strain showed significant treatment effect of CTO-PCI in the entire CTO population $(31.1 \% \pm 9.9 \%$ vs. $34.3 \% \pm 8.7 \%, \mathrm{P}<0.01 ;-17.9 \pm 3.6$ vs. $-19.2 \pm 3.1, \mathrm{P}<0.01)$, and the subgroup with decreased LVEF, infarct size less than $10 \%$, or multi-vessel disease, but not with the 1-vessel disease. In the LAD and LCX CTO territory, radial and circumferential strain showed treatment effect of CTO-PCI on the recovery of strain parameters $(\mathrm{P}<0.01$ for both). In the RCA CTO territory, circumferential and longitudinal strain showed treatment effect of CTO-PCI on the recovery of strain parameters ( $\mathrm{P}<0.05$ for both).

Conclusions: In this single center study, global radial strain and circumferential strain showed treatment effect of successful CTO-PCI at 1-year follow-up in CTOs with the decreased LVEF, infarct size less than $10 \%$, or multi-vessel disease, and the regional strain also showed a similar trend. However, the benefit of CTO-PCI on the strain recovery was not shown in patients with 1-vessel disease. Therefore, whether patients with CTO benefit from PCI still needs further verification.

Keywords: Magnetic resonance; strains; coronary occlusion; percutaneous coronary intervention

Submitted Jul 07, 2021. Accepted for publication Oct 21, 2021.

doi: $10.21037 /$ qims-21-702

View this article at: https://dx.doi.org/10.21037/qims-21-702 


\section{Introduction}

Cardiac magnetic resonance imaging (CMR) is often used to assess left ventricular function and myocardial viability in patients with coronary chronic total occlusion (CTO) as primary outcomes or secondary endpoints in follow-up studies. In recent years, in most of the follow-up studies on CTO, CMR mainly utilized conventional indices such as left ventricular ejection fraction (LVEF), left ventricular end diastolic volume (LVEDV), wall thickening, and semiquantitative techniques for detecting myocardial infarction (MI) (1-3). It, however, remains controversial whether patients with CTO benefit from percutaneous coronary intervention (PCI) (3-5), and more evidence is needed to confirm this aspect. Following the advances in magnetic resonance imaging (MRI) technology, CMR feature tracking (CMR-FT) has become a novel technique for quantifying myocardial deformation by using cine MRI images (6-8). The late gadolinium enhancement (LGE) semiautomated grayscale thresholding technique accurately quantifies the infarct size and thus plays an increasingly strong role in the diagnosis, risk assessment, and prognosis of ischemic heart disease $(2,9,10)$. However, few follow-up studies on CTO-PCI have conducted comprehensive assessment of myocardial deformation and myocardial fibrosis by LGE. Thus, the present study aimed to assess the benefits of PCI in patients with CTO by using CMR-FT and the LGE semiautomated grayscale thresholding technique. We present the following article in accordance with the MDAR checklist (available at https://dx.doi.org/10.21037/qims-21-702).

\section{Methods}

\section{Study population}

From December 2014 to November 2020, 285 patients with CTO were analyzed retrospectively. The inclusion criteria were as follows: (I) age $\geq 18$ years, (II) documented stable angina pectoris, (III) confirmed presence of CTO by coronary angiography, and (IV) performance of CMR. The exclusion criteria were as follows: (I) acute MI within the last 3 months; the term acute $\mathrm{MI}$ is defined as follows: at least one increased cardiac troponin level greater than the 99th percentile upper reference limit; and at least one of the following: myocardial ischemia symptoms, new ischemic changes or pathologic Q waves identified on ECG, imaging results suggesting loss of myocardium or wall motion abnormalities that are ischemic in nature, or coronary thrombus identified on angiography; (II) presence of nonischemic cardiomyopathy; (III) contraindication to MR; (IV) history of allergy to gadolinium contrast agents; (V) Left main artery occlusion; and (VI) renal insufficiency (glomerular filtration rate $<60 \mathrm{~mL} / \mathrm{min} / 1.73 \mathrm{~m}^{2}$ ). We defined CTO as a chronic total occlusion located in the one of three main epicardial arteries or the main side branch with a diameter larger than $2.5 \mathrm{~mm}$. Successful revascularization of CTO was defined as TIMI blood flow of the target vessel reaching level 2 or above. Multi-vessel disease (MVD) was defined as patients with one CTO lesion concurrent with no less than $75 \%$ stenosis in at least one of the other arteries described above. Adverse cardiac events included (I) cardiac death, (II) acute MI, and (III) progressive heart failure requiring hospitalization. The study was conducted in accordance with the Declaration of Helsinki (as revised in 2013). The study protocol was approved by the Ethics Committee of Beijing AnZhen Hospital and written informed consent was obtained from all patients before the study.

For the comparison of strain values, we also retrospectively formed a healthy control group from April 2018 to April 2020 in a heart medical center. This control group consisted 40 subjects (age $52.3 \pm 12.7$ years; male sex, $75 \%$ ), all of which were asymptomatic, with normal ECG, no personal history of cardiopathy, free of cardiovascular disease risk factors and found to have a normal CMR. Written informed consent was obtained from all healthy subjects before the study.

\section{CMR protocol}

CMR was performed before revascularization of the CTO arteries. Clinical follow-up information was obtained at the outpatient clinic where all patients were examined at 6 to 36 months. At 12 months, CMR was performed again. All patients underwent scanning using a $3 \mathrm{~T}$ whole-body scanner (MAGNETOM Verio, A Tim System; Siemens Healthineers, Erlangen, Germany) with a 32-element matrix coil. Cine imaging was performed using a retrospective ECG-gated steady-state free precession technique (imaging parameters: repetition time/echo time/flip angle $=1.5 \mathrm{~ms} / 3.4 \mathrm{~ms} / 50^{\circ}$, time resolution $=41 \mathrm{~ms}$ ).

LGE imaging was performed at least $10 \mathrm{~min}$ after the intravenous administration of $0.2 \mathrm{mmol}$ of gadolinium (Magnevist, Bayer Healthcare, Berlin, Germany) per $\mathrm{kg}$ of body weight by using a two-dimensional (2D) phase-sensitive inversion recovery breath-hold sequence (repetition time/echo time $=4.1 / 1.56 \mathrm{~ms}$, flip angle $=20^{\circ}$, 
field of view $=350 \times 284 \mathrm{~mm}^{2}$ ). Cine cardiac MR imaging and LGE imaging were performed with $8 \mathrm{~mm}$ sections without intersection gaps in short-axis views (from the base to the apex) and views (2- and 4-chamber) of the left ventricle (LV). Cine cardiac MR was also performed in the long-axis 3-chamber view.

\section{CMR strain analysis}

Myocardial strain measurements were performed offline using cvi $^{42}$ (Circle Cardiovascular Imaging, Inc., Calgary, Canada). Left ventricular endocardial and epicardial borders were traced by a threshold-based segmentation method at both end-diastole (defined by the software) and end-systole (defined as the image with the smallest area of the left ventricle) in short- and long-axis cine images (2-chamber, 3 -chamber, and 4-chamber). Trabeculations and papillary muscles were excluded from the ventricular volume. LVEF and LVEDV were measured with the short-axis cine images in the short $3 \mathrm{D}$ module of the software, while the left ventricular strain was measured with short- and longaxis cine images in the tissue tracking module (Figure 1). Peak global radial strain (GRS) and global circumferential strain (GCS) were derived from 2D short-axis acquisitions; peak global longitudinal strain (GLS) was derived from 2D long-axis acquisitions. For regional strain analysis, CMR data were independently analyzed using a 16-segment mode (Bull's eye plot), according to the American Heart Association model.

\section{CMR LGE analysis}

The LV endocardial and epicardial traces on the LGE images were performed manually for determining the myocardial mass, excluding the papillary muscles and intertrabecular blood pool from the total myocardial mass. Normal myocardium was defined visually as a region of myocardium without any apparent LGE on visual inspection. The mean signal intensity and standard deviation (SD) were determined by drawing a region of interest (ROI) in a portion of the normal myocardium (a sample of at least 100 pixels per ROI) on three consecutive midventricular image sections (Figure 2). The mean signal intensities and SDs were averaged across the three midventricular sections to yield an average mean signal intensity and SD. The presence and quantity of LGE were determined with grayscale thresholds of 5 SDs. The total myocardial mass (TMM), total myocardial volume (TMV) of the LV, total enhanced mass (TEM), total enhanced volume (TEV), and the proportion of TEV to TMV (EV\%) in 5 SDs were recorded.

\section{Reproducibility}

Two experienced radiologists analyzed the left ventricular myocardial strain, and LGE images. Twenty cases were analyzed to test interobserver agreement. Intraobserver agreement was analyzed in all 55 cases at intervals of 4 weeks or longer. To measure interobserver and intraobserver agreement, the endocardial and epicardial borders were retraced, and the thresholds were redetermined.

\section{Statistical analysis}

According to the presence of baseline decreased LV ejection fraction (LVEF $<50 \%$ ), we categorized the patients with CTOs into left ventricular ejection fraction decreased (CTOs-LVEFd) group and preserved ejection fraction (CTOs-LVEFp) group. CTOs were also divided into two groups according to the baseline infarct size of $10 \%$ or combined with multi-vessel disease (MVD). Continuous data are reported as mean \pm SD. Baseline characteristics were compared using the independent samples $t$-test or the Mann-Whitney $U$ test for continuous variables and chi-square test for categorical variables. Differences in LVEF, strain, and LGE parameters between follow-up and baseline were compared by paired $t$-test. Differences in LVEF and strain between controls and CTOs were compared by independent $t$-test. Correlations between CMR parameters were assessed using Pearson's correlation coefficient. Intraclass correlation coefficient (ICC) was used for comparing interobserver and intraobserver variability. SPSS statistics (version 21, IBM, Armonk, New York, USA) was used for data analysis. Statistical significance was set at $\mathrm{P}<0.05$.

\section{Results}

From December 2014 to November 2020, 86 of the 285 patients with CTO underwent baseline and follow-up CMR. Among them, 68 patients underwent PCI successfully. Four patients with acute MI within 3 months before PCI, one patient with Left main artery occlusion and eight patients with limited MR images were excluded. Over a median follow-up period of 12 months (range: 8-29 months), 55 patients $(56.9 \pm 11.3$ years, $81.8 \%$ males) with successful 

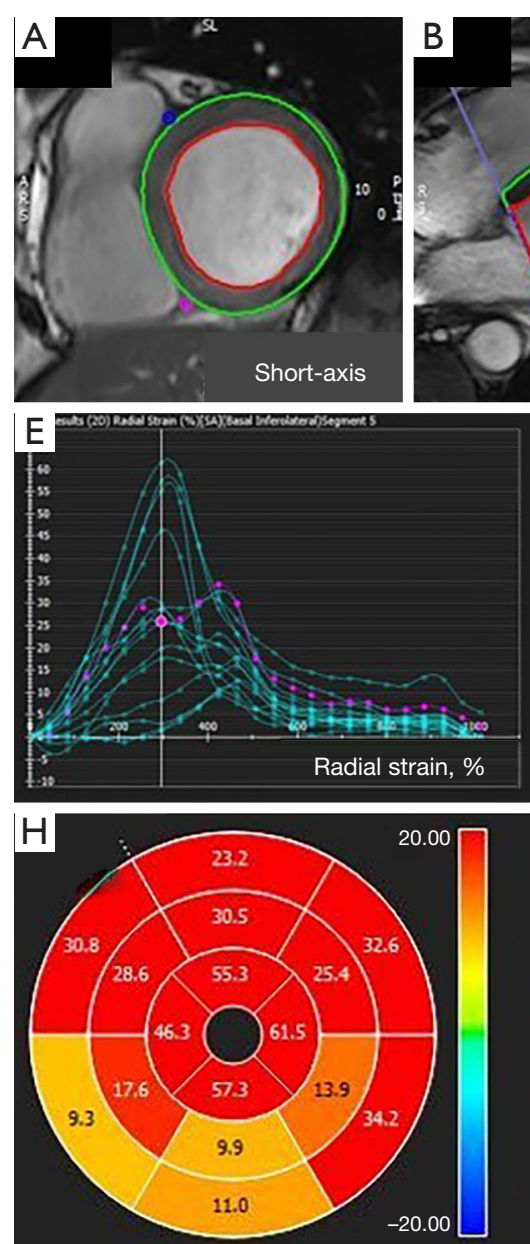

Peak radial strain, \% $96.3 \mathrm{~mm}, \mathrm{AHA}$

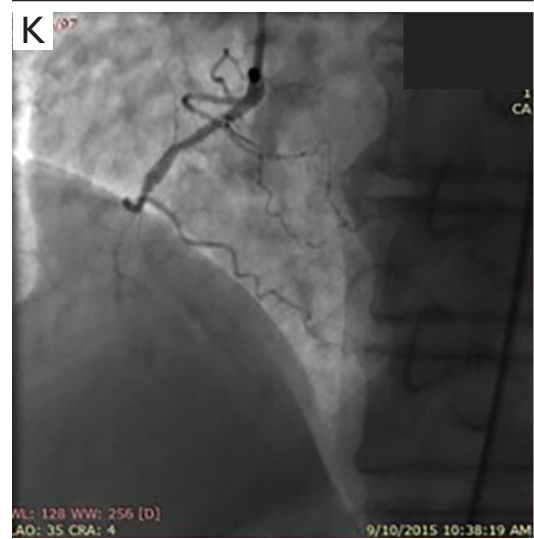

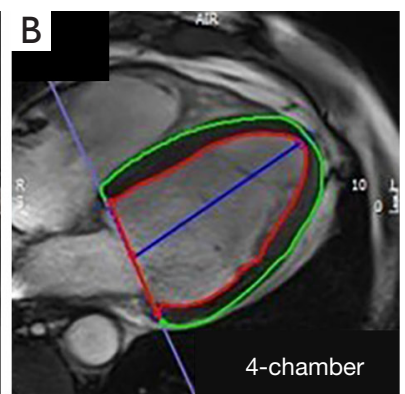
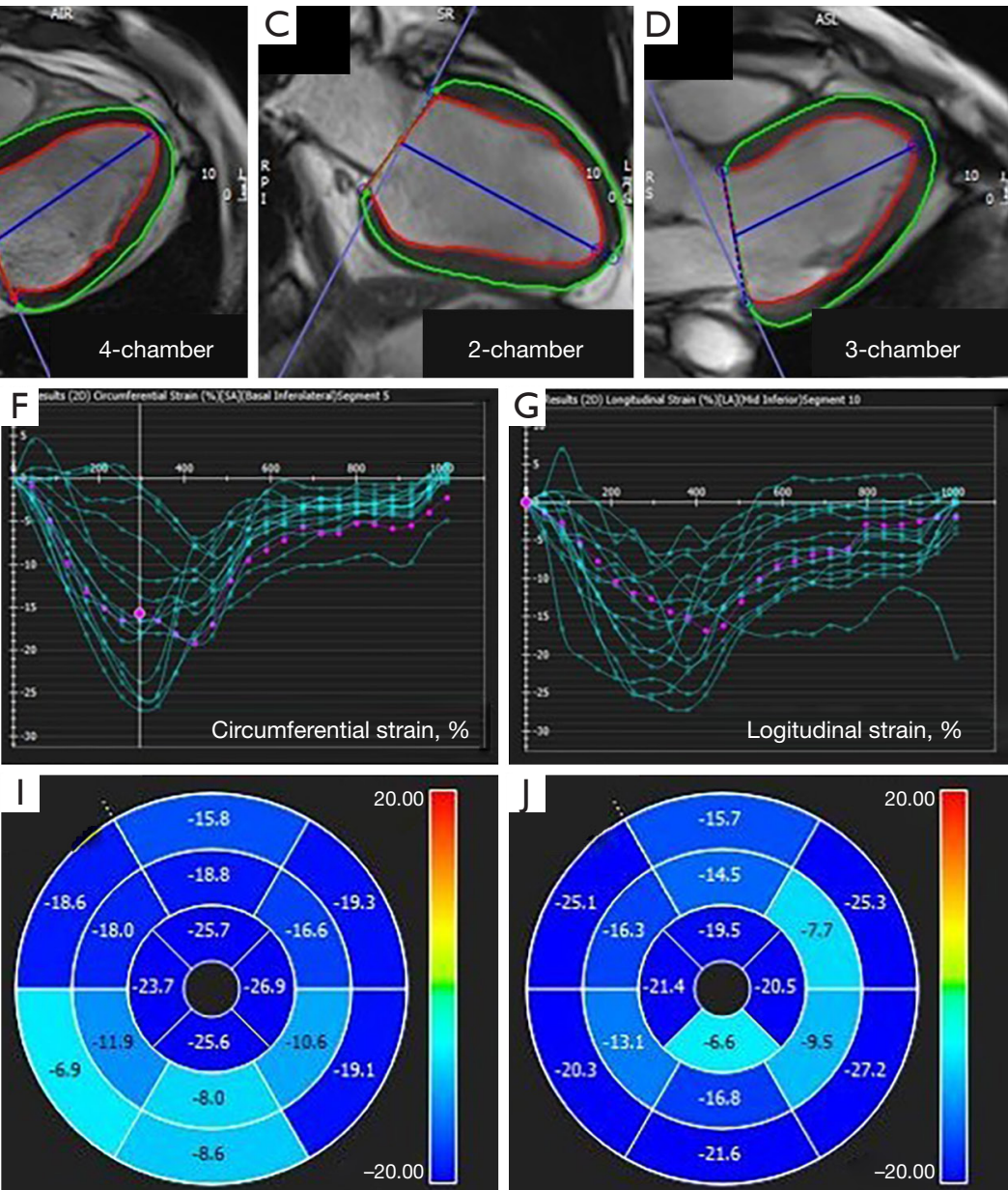

Peak circumferential strain, \% $96.3 \mathrm{~mm}, \mathrm{AHA}$

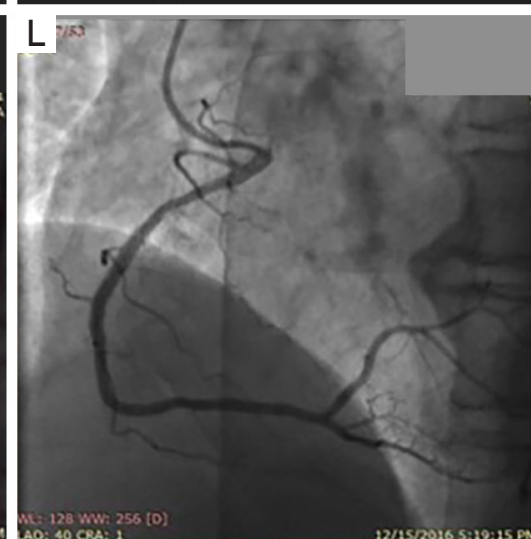

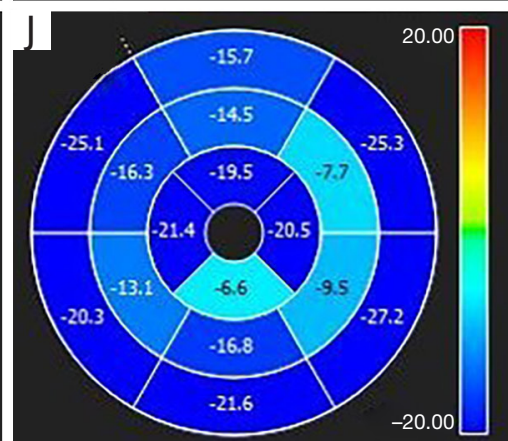

Peak longitudinal strain, \%

$96.3 \mathrm{~mm}, \mathrm{AHA}$

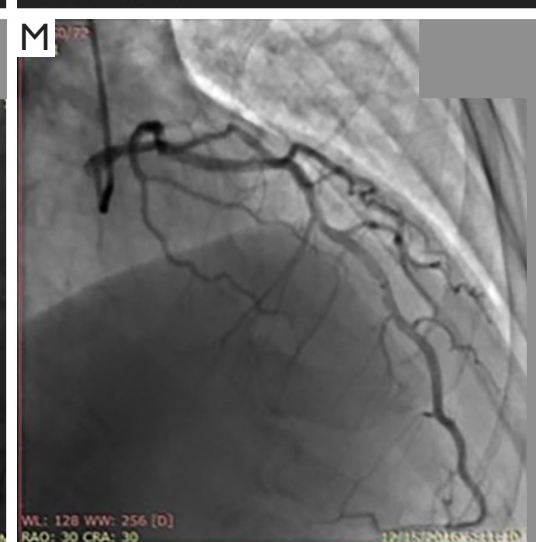

Figure 1 Examples of CMR feature tracking (FT) for quantification of myocardial deformation. (A-D) Cine CMR. Strain curves derived from FT analysis show peak global systolic radial strain (GRS) (E), circumferential strain (GCS) (F), and longitudinal strain (GLS) values (G). (H-J) FT analysis shows regional wall motion abnormality of the basal to mid-septal and inferior wall. (K-M) Coronary angiography showed occlusion of the right coronary artery $(\mathrm{K})$ and successful PCI (L). 

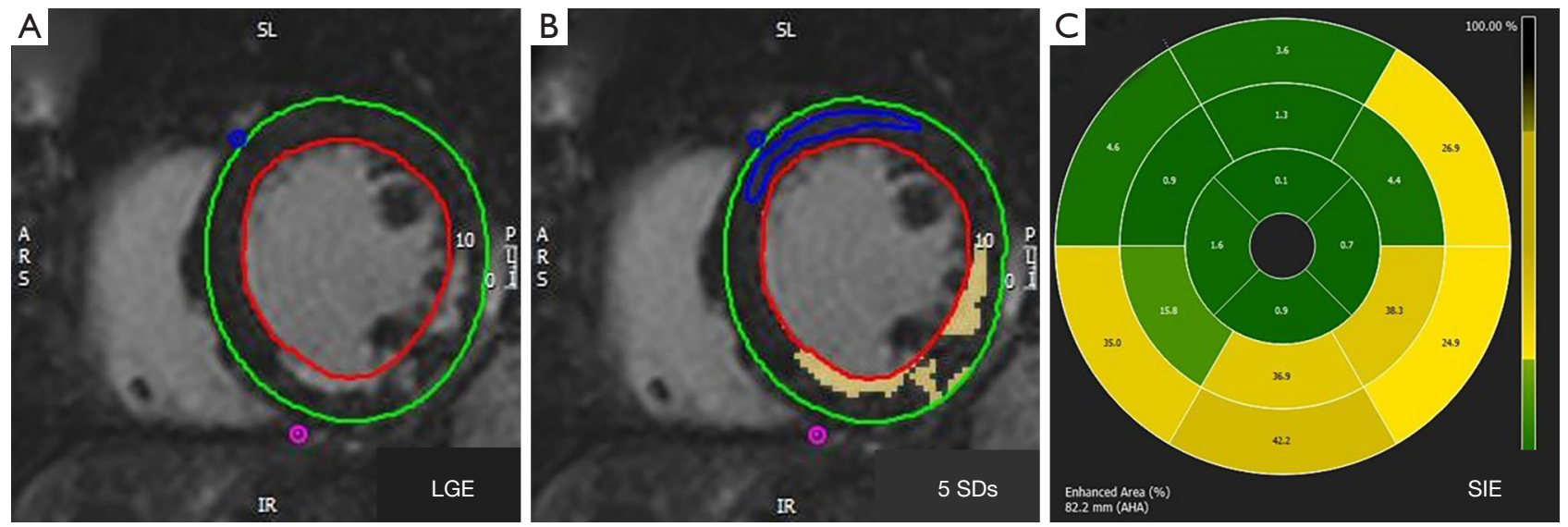

Figure 2 Examples of grayscale thresholding techniques on LGE images. LGE imaging before planimetry (A) and thresholding techniques were performed (B). The 16-segment mode (Bull's eye plot), according to the American Heart Association standard segmentation. The values shown in the 16 segments are the segmental infarct extent $(\mathrm{C})$.

CTO-PCI were included in data analysis. Only one coronary artery occlusion was present in each case in this study, and the main target vessels of CTO were left anterior descending artery (LAD) [n=23 (41.8\%)] and right coronary artery (RCA) [n=24 (43.6\%)]. Thirty-two patients (58.2\%) of CTOs were MVD. In the MVD, the CTO was recanalized, and other significant coronary stenosis was also treated to achieve TIMI blood flow level 2 or above. During 1-year follow-up, no patient experienced adverse cardiac events; however, stent restenosis occurred in two patients.

\section{CMR baseline characteristics}

The global strain values (GRS, GCS, and GLS) by CMR-FT of all 55 CTOs were available at baseline. In the total CTOs population, the mean baseline LVEF was $59.8 \% \pm 11.3 \%$, LVEDV was $118.2 \pm 30.8 \mathrm{~mL}$, TEM was $10.4 \pm 12.4 \mathrm{~g}$, and mean baseline infarct extent was $11.7 \% \pm 10.1 \%$ of the myocardium. Furthermore, $75 \%$ $(41 / 55)$ of patients with CTOs showed no reduction in LVEF ( $>50 \%)$. Compared with the control group, LVEF of CTOs was lower $(\mathrm{P}<0.01)$, while LVEDV was similar $(\mathrm{P}=0.82)$. GRS, GCS, and GLS of CTOs were lower than the control group (all $\mathrm{P}<0.01$ ). Baseline characteristics of the study population are shown in Table 1. GRS, GCS, and GLS were strongly correlated with LVEF $(r=0.82$, $\mathrm{r}=0.83, \mathrm{r}=0.70$, respectively, $\mathrm{P}<0.01$ for all). GRS and GCS were moderately correlated with TEM $(r=-0.44, r=-0.47$, respectively, $\mathrm{P}<0.05$ for both), while GLS was weakly correlated with TEM $(r=-0.24, \mathrm{P}=0.08)$.
For regional strain analysis, 138 segments in LAD CTO territory, 120 segments in RCA CTO territory and 40 segments in left circumflex coronary artery (LCX) CTO territory were analyzed, and the regional strain data of the control group were also collected. The radial strain (RS), circumferential strain (CS) and longitudinal strain (LS) in LAD CTO territory were lower than the control group ( $34.73 \%$ vs. $43.45 \%,-18.72 \%$ vs. $-21.60 \%,-14.14 \%$ vs. $-19.57 \%$, respectively, all $\mathrm{P}<0.01$ ), and the RCA and LCX CTO territory also showed the same trend in each strain index $[(29.67 \%$ vs. $40.10 \%,-16.63 \%$ vs. $-20.53 \%,-12.66 \%$ vs. $-17.24 \%$, respectively, all $\mathrm{P}<0.01),(32.96 \%$ vs. $50.0 \%$, $-18.12 \%$ vs. $-23.85 \%,-16.16 \%$ vs. $-19.06 \%$, respectively, all $\mathrm{P}<0.01)]$.

\section{CTO strain and LGE at follow-up}

Follow-up CMR of all 55 CTOs were available. In the entire CTO population, GRS, GCS, and GLS improved slightly from baseline to 1 year of follow-up, and GRS and GCS showed treatment effect of CTO-PCI on the recovery of global strain parameters $(\triangle \mathrm{GRS}=3.2 \% \pm 8.6 \%, \mathrm{P}<0.01$; $\Delta \mathrm{GCS}=-1.3 \% \pm 3.2 \%, \mathrm{P}<0.01 ; \Delta \mathrm{GLS}=-0.5 \% \pm 2.9 \%$, $\mathrm{P}=0.23)$. LVEF did not improve significantly in the 1 -year follow-up $(59.8 \% \pm 11.3 \%$ vs. $62.0 \% \pm 8.6 \%, \mathrm{P}=0.08)$, and LVEDV did not change significantly $(118.2 \pm 30.8 \mathrm{vs}$. $119.6 \pm 28.8 \mathrm{~mL}, \mathrm{P}=0.68$ ). In the entire CTO population, no significant increase was noted in the TEM and infarct extent from baseline to 1 year of follow-up $(\mathrm{P}>0.05$ for both) (Table 2). GRS, GCS and GLS of follow-up were 
Table 1 Baseline characteristics and CMR findings of CTOs and controls

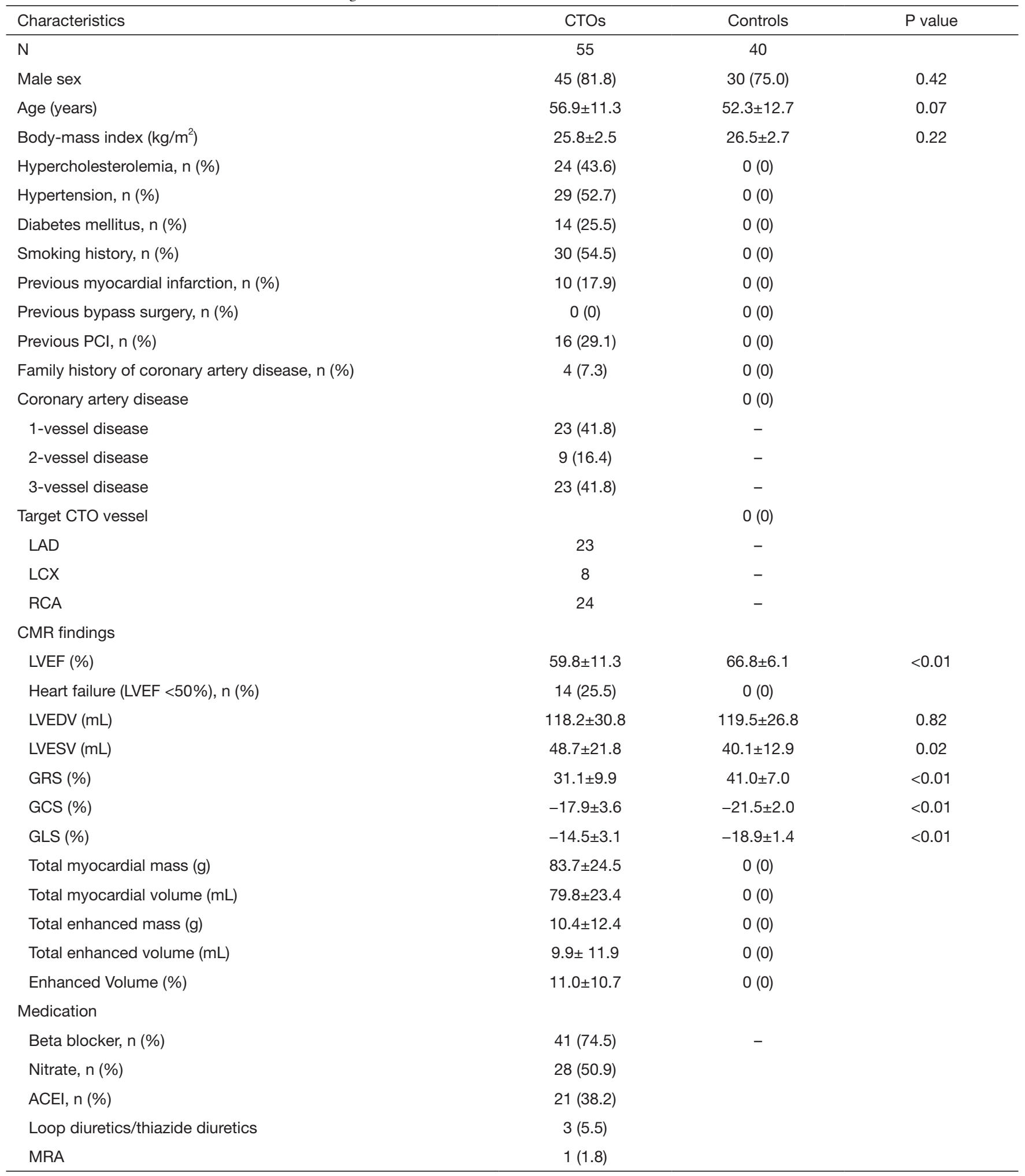

Values are mean \pm SD or $n(\%)$. LVEF, left ventricular ejection fraction; LVEDV, left ventricular end-diastolic volume; LVESV, left ventricular end-systolic volume; GRS, global radial strain; GCS, global circumferential strain; GLS, global longitudinal strain; ACEI, angiotensin converting enzyme inhibitors; MRA, mineralocorticoid receptor antagonist. 
Table 2 Analysis for CMR baseline and flow-up of CTOs

\begin{tabular}{|c|c|c|c|}
\hline Patient-based & Baseline $(n=55)$ & Follow-up $(n=55)$ & $P$ value \\
\hline LVEDV (mL) & $118.2 \pm 30.8$ & $119.6 \pm 28.8$ & 0.68 \\
\hline LVESV (mL) & $48.7 \pm 21.8$ & $48.8 \pm 21.4$ & 0.95 \\
\hline GRS (\%) & $31.1 \pm 9.9$ & $34.3 \pm 8.7$ & 0.009 \\
\hline GLS (\%) & $-14.5 \pm 3.1$ & $-14.9 \pm 2.9$ & 0.23 \\
\hline Total myocardial mass (g) & $83.7 \pm 24.5$ & $85.0 \pm 23.4$ & 0.58 \\
\hline Total myocardial volume $(\mathrm{mL})$ & $79.8 \pm 23.4$ & $81.0 \pm 22.3$ & 0.58 \\
\hline Total enhanced mass (g) & $10.4 \pm 12.4$ & $11.4 \pm 12.2$ & 0.29 \\
\hline
\end{tabular}

Values are mean \pm SD or $n(\%)$. LVEF, left ventricular ejection fraction; LVEDV, left ventricular end-diastolic volume; LVESV, left ventricular end-systolic volume; GRS, global radial strain; GCS, global circumferential strain; GLS, global longitudinal strain.

moderately correlated with TEM ( $\mathrm{r}=-0.38, \mathrm{r}=-0.42$, $\mathrm{r}=-0.39$, respectively, all $\mathrm{P}<0.05$ ).

In the LAD and LCX CTO territory, RS and CS improved slightly from baseline to 1 year of follow-up, and showed treatment effect of CTO-PCI on the recovery of strain parameters $[(\Delta \mathrm{RS}=4.85 \% \pm 16.68 \%, \mathrm{P}<0.01 ; \Delta \mathrm{CS}$ $=1.64 \% \pm 5.05 \%, \mathrm{P}<0.01 ; \Delta \mathrm{LS}=-0.63 \% \pm 12.38 \%, \mathrm{P}=0.55)$, $(\Delta \mathrm{RS}=6.70 \% \pm 17.97 \%, \mathrm{P}=0.02 ; \Delta \mathrm{CS}=2.41 \% \pm 6.42 \%$, $\mathrm{P}=0.02 ; \Delta \mathrm{LS}=-0.79 \% \pm 10.32 \%, \mathrm{P}=0.64)$, respectively]. In the RCA CTO territory, RS, CS and LS improved slightly from baseline to 1 year of follow-up, and CS and LS showed treatment effect of CTO-PCI on the recovery of strain parameters $(\Delta \mathrm{RS}=2.40 \% \pm 14.0 \%, \mathrm{P}=0.06 ; \Delta \mathrm{CS}$ $=1.07 \% \pm 5.60 \%, \mathrm{P}=0.04 ; \Delta \mathrm{LS}=2.0 \% \pm 10.58 \%, \mathrm{P}=0.04)$.

\section{Subgroup analysis}

Fourteen CTOs showed reduction in LVEF (<50\%) and 41 CTOs showed preserved ejection fraction at baseline. The LVEF and LVEDV of CTO-LVEFp group were not significantly different from the control group at baseline (P>0.05 for both), but the GRS, GCS, and GLS of CTOLVEFp group at baseline were lower than those of the control group $(\mathrm{P}<0.01$ for all) (Table 3, Figure 3). The LVEF, GRS, GCS, and GLS of CTO-LVEFd group at baseline were lower than those of the CTO- LVEFp group $(\mathrm{P}<0.01$ for all). After PCI, the number of patients with decreased left ventricular function decreased from 14 at baseline to 5 in the 1-year follow-up. GRS and GCS of CTO-LVEFd group showed treatment effect of CTOPCI on the recovery of global strain parameters ( $\triangle$ GRS $=7.0 \% \pm 8.1 \%, \mathrm{P}<0.01 ; \Delta \mathrm{GCS}=-3.1 \% \pm 3.0 \%, \mathrm{P}<0.01 ; \Delta \mathrm{GLS}$ $=-1.1 \% \pm 3.3 \%, \mathrm{P}=0.25$ ), and LVEF of CTO-LVEFd group improved significantly in the 1 -year follow-up $(\mathrm{P}<0.01)$ (Figure 3). But in the CTO-LVEFp group, no significant increase was noted in the LVEF, GRS, GCS, and GLS from baseline to 1 year of follow-up ( $\mathrm{P}>0.05$ for all). Medication use among CTO-LVEFd group was similar with those among CTO-LVEFp group $(\mathrm{P}>0.05)$ (Table S1).

In the subgroup analysis, 23 CTOs had an infarct size greater than $10 \%$ (CTO-EVg) and 32 CTOs had an infarct size lower than 10\% (CTO-EVl). The LVEF, GRS, GCS, and GLS of the two group at baseline were lower than those of the control group $(\mathrm{P}<0.05$ for all) (Table 3). However, the LVEDV of these two groups did not increase significantly compared with the control group. There was no significant difference in LVEF, LVEDV, GRS, GCS and GLS between the two groups at baseline. GRS and GCS of CTO-EVl group showed treatment effect of CTOPCI on the recovery of global strain parameters $(\triangle \mathrm{GRS}$ $=3.7 \% \pm 9.3 \%, \mathrm{P}=0.03 ; \Delta \mathrm{GCS}=-1.5 \% \pm 3.2 \%, \mathrm{P}=0.02$; $\Delta \mathrm{GLS}=-0.9 \% \pm 3.0 \%, \mathrm{P}=0.12)$. In the CTO-EVg group, no significant increase was noted in the LVEF, LVEDV, GRS, GCS and GLS from baseline to 1 year of follow-up $(\mathrm{P}>0.05$ for all) (Figure 4).

Thirty-two patients (58.2\%) of CTOs were MVD and 23 
Table 3 Subgroup analysis for the LVEF and LGE of CTOs

\begin{tabular}{|c|c|c|c|c|c|c|}
\hline Variables & $\operatorname{LVEF}_{p}(\mathrm{n}=41)$ & $\operatorname{LVEF}_{d}(\mathrm{n}=14)$ & $\mathrm{P}$ value & $E V<10 \%(n=32)$ & $E V \geq 10 \%(n=23)$ & $P$ value \\
\hline \multicolumn{7}{|l|}{ LVEF (\%) } \\
\hline Baseline & $64.9 \pm 7.9$ & $44.8 \pm 3.4$ & $<0.01$ & $62.0 \pm 11.6$ & $56.8 \pm 10.2$ & 0.09 \\
\hline 1-year follow-up & $64.5 \pm 6.5$ & $54.8 \pm 9.9$ & $<0.01$ & $63.4 \pm 7.6$ & $60.1 \pm 9.5$ & 0.15 \\
\hline Change in LVEF & $-0.43 \pm 7.2$ & $10.0 \pm 10.8$ & $<0.01$ & $1.5 \pm 10.2$ & $3.3 \pm 8.0$ & 0.48 \\
\hline Baseline & $114.8 \pm 31.8$ & $128.1 \pm 26.2$ & 0.16 & $111.2 \pm 28.4$ & $128.0 \pm 31.8$ & 0.05 \\
\hline 1-year follow-up & $117.7 \pm 31.1$ & $125.1 \pm 20.6$ & 0.41 & $116.0 \pm 29.1$ & $124.4 \pm 28.3$ & 0.29 \\
\hline Change in LVEDV & $2.9 \pm 24.1$ & $-3.0 \pm 25.5$ & 0.45 & $4.9 \pm 27.8$ & $-3.5 \pm 18.0$ & 0.21 \\
\hline \multicolumn{7}{|l|}{ GRS (\%) } \\
\hline Change in GRS & $1.9 \pm 8.5$ & $7.0 \pm 8.1$ & 0.05 & $3.7 \pm 9.3$ & $2.4 \pm 7.7$ & 0.58 \\
\hline \multicolumn{7}{|l|}{ GCS (\%) } \\
\hline Baseline & $-19.1 \pm 3.1$ & $-14.1 \pm 2.2$ & $<0.01$ & $-18.6 \pm 3.7$ & $-16.8 \pm 3.4$ & 0.08 \\
\hline 1-year follow-up & $-19.9 \pm 2.8$ & $-17.2 \pm 3.1$ & $<0.01$ & $-20.1 \pm 2.9$ & $-17.9 \pm 2.9$ & 0.01 \\
\hline Change in GCS & $-0.7 \pm 3.0$ & $-3.1 \pm 3.0$ & 0.02 & $-1.5 \pm 3.2$ & $-1.1 \pm 3.1$ & 0.66 \\
\hline \multicolumn{7}{|l|}{ GLS (\%) } \\
\hline Baseline & $-15.3 \pm 2.9$ & $-12.1 \pm 2.7$ & $<0.01$ & $-14.8 \pm 3.2$ & $-14.0 \pm 3.0$ & 0.35 \\
\hline 1-year follow-up & $-15.5 \pm 2.7$ & $-13.2 \pm 2.7$ & $<0.01$ & $-15.7 \pm 2.8$ & $-13.9 \pm 2.8$ & 0.03 \\
\hline \multicolumn{7}{|l|}{ Enhanced volume (\%) } \\
\hline Baseline & $5.4 \pm 4.4$ & $21.4 \pm 8.5$ & $<0.001$ & $4.9 \pm 2.9$ & $21.5 \pm 9.3$ & $<0.01$ \\
\hline 1-year follow-up & $6.3 \pm 6.2$ & $20.6 \pm 10.3$ & $<0.001$ & $6.5 \pm 6.1$ & $19.6 \pm 10.6$ & $<0.01$ \\
\hline Change in EV & $1.1 \pm 6.2$ & $-0.2 \pm 9.0$ & 0.68 & $2.6 \pm 4.8$ & $-1.7 \pm 8.7$ & 0.04 \\
\hline
\end{tabular}

Values are mean $\pm S D$. $L V E F_{d}$, left ventricular ejection fraction decreased; EV, enhanced volume; LVEF , left ventricular ejection fraction preserved; TEM, total enhanced mass; GRS, global radial strain; GCS, global circumferential strain; GLS, global longitudinal strain.

patients were 1-vessel disease. The baseline GRS, GCS, and GLS of two groups were lower than those of the control group $(\mathrm{P}<0.01$ for all), which were similar in both group ( $\mathrm{P}>0.05$ for all) (Table 4). The baseline LVEF of 1-vessel disease was similar with the control group $(\mathrm{P}=0.26)$, while the baseline LVEF of MVD was lower than the control group $(\mathrm{P}<0.01)$. The baseline LVEDV of two groups were similar with the control group ( $>0.05$ for both). The baseline LVEF of MVD were lower than 1-vessel disease, while global strain parameters were similar ( $\mathrm{P}>0.05$ for all). GRS and GCS of MVD group showed treatment effect of CTO-PCI on the recovery of global strain parameters 


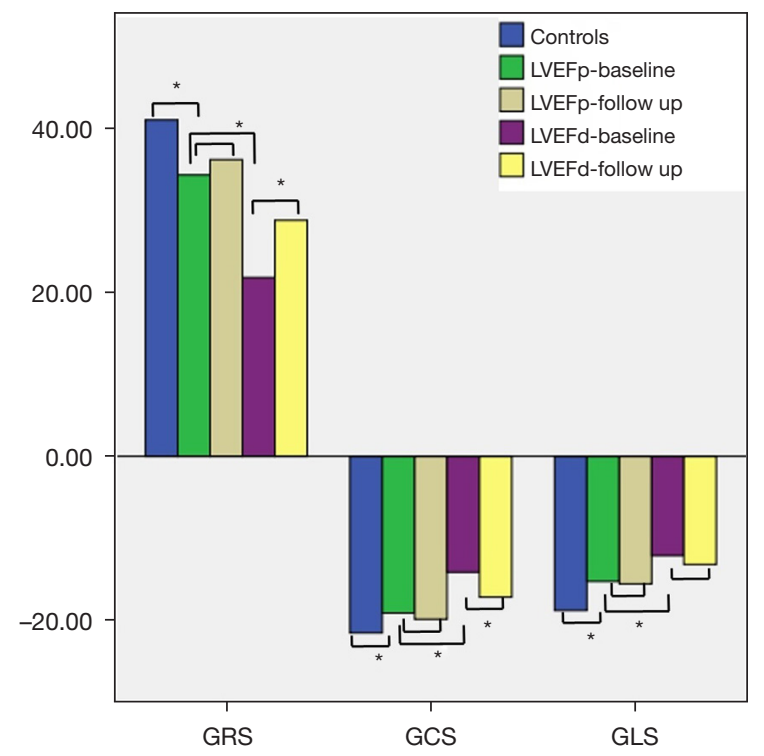

Figure 3 Comparison of left ventricular global strain parameters between baseline and 1-year follow-up based on the presence of baseline decreased LV ejection fraction (LVEF <50\%). LVEFp, CTOs with preserved left ventricular ejection fraction; LVEFd, CTOs with decreased left ventricular ejection fraction; GRS, peak global radial strain (\%); GCS, peak global circumferential strain (\%); GLS, peak global longitudinal strain (\%); ${ }^{*}, \mathrm{P}<0.05$.

$(\Delta \mathrm{GRS}=-4.4 \% \pm 8.5 \%, \mathrm{P}<0.01 ; \Delta \mathrm{GCS}=-1.8 \% \pm 3.1 \%$, $\mathrm{P}<0.01 ; \Delta \mathrm{GLS}=-0.7 \% \pm 2.6 \%, \mathrm{P}=0.15)$, and LVEF also showed improvement $(\mathrm{P}=0.02)$. In the 1 -vessel disease, no significant increase was noted in the LVEF, GRS, GCS, and GLS from baseline to 1 year of follow-up ( $\mathrm{P}>0.05$ for all) (Figure 5).

\section{Interobserver and intraobserver agreement}

Intraobserver reproducibility was excellent between the first and second evaluations for three global strain parameters (ICC $=0.88-0.94, \mathrm{P}<0.01$ ). Interobserver reproducibility was also excellent between two observers for three global strain parameters (ICC $=0.80-0.95, \mathrm{P}<0.01$ ). For the regional strain parameters, the intraobserver reproducibility of regional strains of the LAD, LCX, and RCA territory were excellent in RS and CS (ICC $=0.83-0.91, \mathrm{P}<0.01$ ), and the intraobserver reproducibility were good in LS (ICC $=0.56-$ $0.80, \mathrm{P}<0.01)$; the interobserver reproducibility between two observers for regional strains of the LAD, LCX, and RCA territory were also excellent in RS and CS (ICC

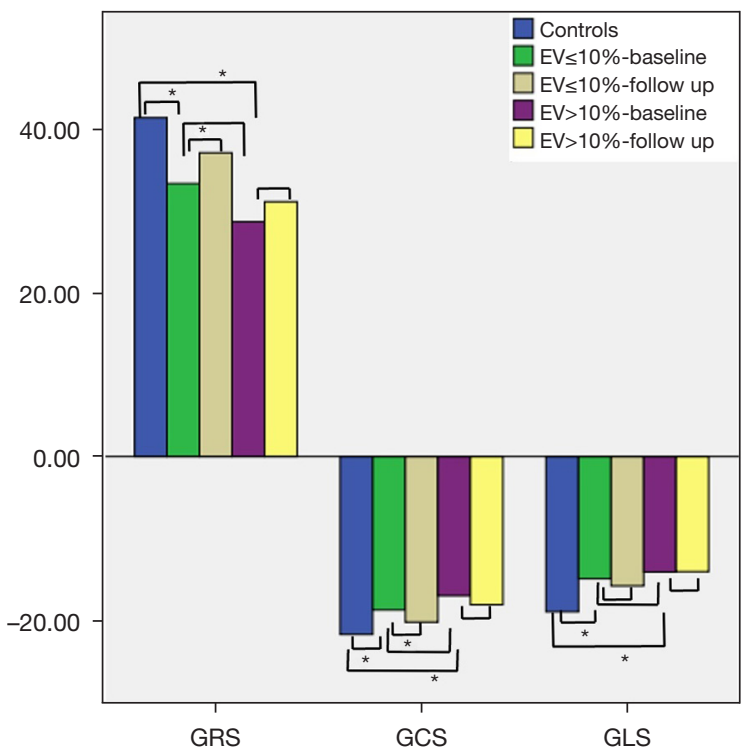

Figure 4 Comparison of left ventricular global strain parameters between baseline and 1-year follow-up based on the infarct size (EV $\leq 10 \%)$. EV, enhanced volume; GRS, peak global radial strain (\%); GCS, peak global circumferential strain (\%); GLS, peak global longitudinal strain (\%); *, $\mathrm{P}<0.05$.

$=0.88-0.91, \mathrm{P}<0.01)$, and the interobserver reproducibility was good in LS (ICC $=0.59-0.81, \mathrm{P}<0.01)$.

Intraobserver agreement was good with the $5 \mathrm{SDs}$ thresholding method in all patients (ICC $=0.94, \mathrm{P}<0.01$ ). Interobserver agreement was also good with the $5 \mathrm{SDs}$ thresholding method in 20 patients (ICC $=0.87, \mathrm{P}<0.01$ ).

\section{Discussion}

The main findings of the present study are as follows: (I) three quarters of CTOs showed preserved ejection fraction and no enlargement of left ventricle at baseline, but the global strains were lower than the controls; (II) In the total CTOs population, global strains improved over time, and GRS and GCS showed a significant treatment effect of successful CTO-PCI; (III) GRS and GCS of CTO-LVEFd and CTO-EVl group showed treatment effect of CTOPCI on the recovery of global strain parameters; (IV) in the MVD, when the CTO and the other significant coronary stenosis were recanalized, GRS and GCS showed treatment effect of CTO-PCI on the recovery of global strain 
Table 4 Subgroup analysis for the Multi-vessel and CMR strain of CTOs

\begin{tabular}{|c|c|c|c|}
\hline Variables & 1 -vessel $(n=23)$ & Multi-vessel $(n=32)$ & $P$ value \\
\hline Baseline & $64.3 \pm 9.7$ & $56.6 \pm 11.3$ & 0.01 \\
\hline 1-year follow-up & $63.1 \pm 8.9$ & $61.3 \pm 8.4$ & 0.44 \\
\hline Change in LVEF & $-1.2 \pm 6.5$ & $4.7 \pm 10.3$ & 0.02 \\
\hline Baseline & $112.4 \pm 28.2$ & $122.4 \pm 32.2$ & 0.24 \\
\hline 1-year follow-up & $114.3 \pm 24.3$ & $123.4 \pm 31.5$ & 0.25 \\
\hline Change in LVEDV & $1.9 \pm 20.5$ & $1.0 \pm 27.1$ & 0.90 \\
\hline \multicolumn{4}{|l|}{ GRS (\%) } \\
\hline Change in GRS & $1.5 \pm 8.7$ & $4.4 \pm 8.5$ & 0.21 \\
\hline \multicolumn{4}{|l|}{ GCS (\%) } \\
\hline Baseline & $-18.9 \pm 3.2$ & $-17.1 \pm 3.8$ & 0.08 \\
\hline 1-year follow-up & $-19.6 \pm 2.9$ & $-18.9 \pm 3.2$ & 0.44 \\
\hline Change in GCS & $-0.7 \pm 3.2$ & $-1.8 \pm 3.1$ & 0.22 \\
\hline \multicolumn{4}{|l|}{ GLS (\%) } \\
\hline Baseline & $-15.3 \pm 2.9$ & $-13.9 \pm 3.2$ & 0.10 \\
\hline 1-year follow-up & $-15.5 \pm 2.7$ & $-14.5 \pm 3.0$ & 0.23 \\
\hline \multicolumn{4}{|l|}{ Enhanced volume (\%) } \\
\hline Baseline & $12.6 \pm 10.7$ & $10.3 \pm 10.9$ & 0.44 \\
\hline 1-year follow-up & $11.9 \pm 8.5$ & $11.9 \pm 11.6$ & 1.0 \\
\hline Change in EV & $-0.2 \pm 6.1$ & $1.6 \pm 7.4$ & 0.37 \\
\hline
\end{tabular}

Values are mean \pm SD or $n$. TEM, total enhanced mass; EV, enhanced volume; GRS, global radial strain; GCS, global circumferential strain; GLS, global longitudinal strain.

parameters; (V) in the CTO territory, the regional analysis showed treatment effect of CTO-PCI on the recovery of strain parameters; (VI) in the 1-vessel disease, no strain treatment effect of CTO-PCI was noted in the 1 year of follow-up.

CMR-FT technology can be applied to routine cine images, without additional pulse sequences. It is widely used in the diagnosis of various cardiovascular diseases, including ischemic heart disease. Previous studies have demonstrated an excellent intra- and interobserver reproducibility as well as a good interstudy agreement (11-13). Our present study also showed similar results. There is no unanimous 


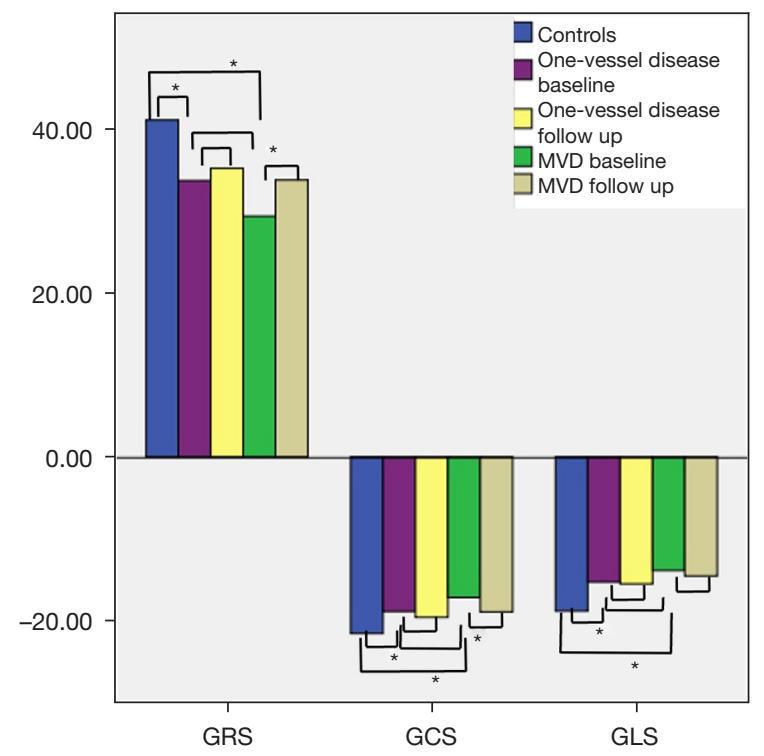

Figure 5 Comparison of left ventricular strain parameters between baseline and1-year follow-up based on multi-vessel disease. MVD, multi-vessel disease; GRS, peak global radial strain (\%); GCS, peak global circumferential strain (\%); GLS, peak global longitudinal strain $(\%) ;{ }^{*}, \mathrm{P}<0.05$.

opinion on the accuracy of CMR-FT 2D and 3D technique for measuring myocardial strain. A previous study showed that the $2 \mathrm{D}$ technique is more reliable and repeatable (11); hence, $2 \mathrm{D}$ strain parameters were used in the present study. It should be noted that GCS and GLS has better reproducibility than GRS. Normal values of GCS, GLS, and GRS range from $-17 \%$ to $-25 \%$, from $-16 \%$ to $-21 \%$, and from $35 \%$ to $51 \%$, respectively $(6,14)$. The strain values of our healthy control group (GCS=-22\%, GLS $=-19 \%$, GRS $=41 \%$ ) were also consistent with the previous reports.

LVEF reflects the global function of the LV, but it cannot distinguish the regional myocardial strain in different directions, and it cannot reflect the early damage caused by heart disease. CMR-FT technology provides an incremental value for risk stratification in various cardiac diseases (15-17). Some studies have shown that feature tracking-derived GLS is a powerful independent predictor of adverse outcomes in patients with reduced ejection fraction (18-20). An important predictive value of GLS was also shown in another multicenter study of patients with preserved ejection fraction, incremental to common clinical and imaging risk factors (age, body mass index, diabetes, hypertension, heart rate, diastolic blood pressure, left ventricular end-diastolic volume index, left ventricular ejection fraction, left atrial volume, LGE, right ventricular ejection fraction) (21). This previous study also showed that patients with GLS $\geq$ median $(-20 \%)$ had significantly reduced event-free survival as compared to those with GLS $<$ median. In our present study, most of the CTOs (75\%) had no reduction in LVEF (>50\%), but the mean GLS at baseline $(-14.5 \%)$ and 1 -year follow-up $(-14.9 \%)$ was lower than the controls without significant improvement after PCI. In the subgroup analysis, there was no significant improvement was noted in GLS. Therefore, although most of CTOs with preserved ejection fraction in the present study and no major cardiovascular events occurred during the 1-year follow-up, GLS decreased and did not improve after successful PCI, which reflected the early damage of cardiac function and may be considered as a potential risk factor.

The independent prognostic value of GCS is controversial. The baseline GCS $(-18 \%)$ of CTOs in our group was lower than the healthy controls $(22 \%)$ in our study and previously reported in healthy population $(8,22)$. GCS in our study showed treatment effect of CTO-PCI on the recovery of global strain parameters, especially for the subgroup with decreased LVEF, infarct size less than $10 \%$, and MVD. A study on tagging also showed that GCS has incremental independent prognostic value in addition to the clinical variables of LVEF and LGE (23). Mangion et al. showed that circumferential strain has independent prognostic importance in study participants with acute STsegment elevation myocardial infarction (24). However, other follow-up studies of acute MI did not support this finding (25-27). Thus, more evidence needs to be provided in the future.

GRS, GCS and GLS of CTOs were significantly decreased compared with the control group, and the decrease of GRS and GCS was more obvious in the subgroup with myocardial infarction size greater than $10 \%$, which was consistent with the pathophysiological characteristics of ischemic heart disease. Myocardial ischemic infarction generally involved subendocardium first, then to the middle and epicardium of myocardium. Circumferential myofibers are located within the midwall; therefore, transmural MI or larger infarct size is more likely to cause the dysfunction of GCS. We also found that CTO with small infarct size $(\mathrm{EV}<10 \%)$ is more likely to benefit from GCS functional recovery after successful PCI. Longitudinal myofibers are distributed within the subendocardium of the heart; therefore, it is more susceptible to myocardial ischemia or MI caused by epicardial artery stenosis. Despite successful 
revascularization of CTO, GLS did not improve even in small infarct size in our study.

A recent study on fast-strain encoded magnetic resonance (fast-SENC) showed that the \% normal myocardium, determined by fast-SENC, enables improved identification of asymptomatic patients with subclinical $\mathrm{LV}$ dysfunction compared with LV ejection fraction and risk stratification of patients with so far asymptomatic heart failure (28). They used Cutoff value of $\leq-17 \%$ for "normal" myocardium, and measured the total number of segments per patient with LS or CS $\leq-17 \%$. In our study, the analysis based on CMR baseline data shows that three quarters of CTOs showed preserved ejection fraction and no enlargement of left ventricle at baseline, but the global strains were lower than the controls, may also indicating early damage to left ventricular function or subclinical $\mathrm{LV}$ dysfunction. The decrease of the regional strains in CTO territory were lower than the control group also indicates that coronary artery occlusion has caused changes in regional myocardial mechanics. In the entire CTO population, GRS, GCS, and GLS improved slightly from baseline to 1 year of followup, and GRS and GCS showed treatment effect of CTOPCI on the recovery of global strain parameters. The regional strains in CTO territory also showed the slightly improvement. But LVEF did not improve in the 1-year follow-up in the entire CTO population. This may indicate that there is a subclinical improvement in left ventricular function.

The CMR LGE technique is routinely used in clinical work. It can accurately quantify infarcted myocardium and evaluate the prognosis of heart disease. For patients with ischemic heart disease or nonischemic heart disease, the larger the area of LGE in CMR, the worse is the prognosis $(29,30)$. A study also showed that when the myocardial ischemic area of coronary heart disease is greater than $10-12.5 \%$ (31), PCI is better than medical therapy. Therefore, this study tried to use $10 \%$ of the infarct size as the subgroup classification basis. A previous study showed that semiautomated LGE CMR grayscale thresholding with 5 SDs yields the strongest correlation of the extent of LGE identified with PET and is highly reproducible in patients with CTO (9). In the present study, the baseline TEM was small in CTOs, which is consistent with other previous studies $(32,33)$.

\section{Conclusions}

In conclusion, in this small-sample single center study, GRS and GCS showed treatment effect of successful CTO-PCI at 1-year follow-up in CTOs with the decreased LVEF, infarct size less than $10 \%$, or MVD, and the regional strain also showed a similar trend. However, the strain treatment effect of CTO-PCI was not shown in patients with 1-vessel disease. Therefore, whether patients with CTO benefit from PCI still needs further verification.

\section{Study limitations}

Our study has some limitations. This is a single center study with limited number of patients. The non-PCI group of CTOs was not included in this study because the number of patients with 1-year MRI follow-up was too small. Although there was no significant difference in age and gender between the CTO group and the control group, the age was not fully matched because there were fewer healthy people over 65 years old. Patients with single vessel CTO may not profit in terms of strain recovery but may profit in terms of reduction in ischemic area or clinical symptoms. In the future, we will increase the sample size, evaluate myocardial ischemia, clinical symptoms to further confirm the results of the present research.

\section{Acknowledgments}

We acknowledge Hangzhou CVIC Smart Technology Co., Ltd. for their assistance.

Funding: This work was supported by National Natural Science Foundation of China (81671650), National Natural Science Foundation of China (81971569), Capital Health Development Research Project (2018-2-2063) and Beijing Municipal Education Commission Science and Technology Plan (KM202010025016).

\section{Footnote}

Reporting Checklist: The authors have completed the MDAR checklist. Available at https://dx.doi.org/10.21037/qims21-702

Conflicts Interest: All authors have completed the ICMJE uniform disclosure form (available at https://dx.doi. org/10.21037/qims-21-702). The authors have no conflicts of interest to declare.

Ethical Statement: The authors are accountable for all aspects of the work in ensuring that questions related 
to the accuracy or integrity of any part of the work are appropriately investigated and resolved. The study was conducted in accordance with the Declaration of Helsinki (as revised in 2013). The study protocol was approved by the Ethics Committee of Beijing AnZhen Hospital and written informed consent was obtained from all patients before the study.

Open Access Statement: This is an Open Access article distributed in accordance with the Creative Commons Attribution-NonCommercial-NoDerivs 4.0 International License (CC BY-NC-ND 4.0), which permits the noncommercial replication and distribution of the article with the strict proviso that no changes or edits are made and the original work is properly cited (including links to both the formal publication through the relevant DOI and the license). See: https://creativecommons.org/licenses/by-nc-nd/4.0/.

\section{References}

1. Danek BA, Karatasakis A, Tajti P, Sandoval Y, Karmpaliotis D, Alaswad K, et al. Incidence, Treatment, and Outcomes of Coronary Perforation During Chronic Total Occlusion Percutaneous Coronary Intervention. Am J Cardiol 2017;120:1285-92.

2. Elias J, van Dongen IM, Råmunddal T, Laanmets $\mathrm{P}$, Eriksen E, Meuwissen M, Michels HR, Bax M, Ioanes D, Suttorp MJ, Strauss BH, Barbato E, Marques KM, Claessen BEPM, Hirsch A, van der Schaaf RJ, Tijssen JGP, Henriques JPS, Hoebers LP; EXPLORE investigators. Long-term impact of chronic total occlusion recanalisation in patients with ST-elevation myocardial infarction. Heart 2018;104:1432-8.

3. Henriques JP, Hoebers LP, Råmunddal T, Laanmets P, Eriksen E, Bax M, Ioanes D, Suttorp MJ, Strauss BH, Barbato E, Nijveldt R, van Rossum AC, Marques KM, Elias J, van Dongen IM, Claessen BE, Tijssen JG, van der Schaaf RJ; EXPLORE Trial Investigators. Percutaneous Intervention for Concurrent Chronic Total Occlusions in Patients With STEMI: The EXPLORE Trial. J Am Coll Cardiol 2016;68:1622-32.

4. Lee SW, Lee PH, Ahn JM, Park DW, Yun SC, Han S, et al. Randomized Trial Evaluating Percutaneous Coronary Intervention for the Treatment of Chronic Total Occlusion. Circulation 2019;139:1674-83.

5. Grün S, Schumm J, Greulich S, Wagner A, Schneider S, Bruder O, Kispert EM, Hill S, Ong P, Klingel K, Kandolf R, Sechtem U, Mahrholdt H. Long-term follow- up of biopsy-proven viral myocarditis: predictors of mortality and incomplete recovery. J Am Coll Cardiol 2012;59:1604-15.

6. Claus P, Omar AMS, Pedrizzetti G, Sengupta PP, Nagel E. Tissue Tracking Technology for Assessing Cardiac Mechanics: Principles, Normal Values, and Clinical Applications. JACC Cardiovasc Imaging 2015;8:1444-60.

7. Andre F, Steen H, Matheis P, Westkott M, Breuninger K, Sander Y, Kammerer R, Galuschky C, Giannitsis E, Korosoglou G, Katus HA, Buss SJ. Age- and genderrelated normal left ventricular deformation assessed by cardiovascular magnetic resonance feature tracking. J Cardiovasc Magn Reson 2015;17:25.

8. Vo HQ, Marwick TH, Negishi K. MRI-Derived Myocardial Strain Measures in Normal Subjects. JACC Cardiovasc Imaging 2018;11:196-205.

9. Zhang LJ, Dong W, Li JN, Mi HZ, Jiao J, Dou RY, An J, Liu JL, He Y, Song XT. Quantification of late gadolinium enhancement cardiovascular MRI in patients with coronary artery chronic total occlusion. Clin Radiol 2020;75:643. e19-26.

10. Bulluck H, Hammond-Haley M, Weinmann S, MartinezMacias R, Hausenloy DJ. Myocardial Infarct Size by CMR in Clinical Cardioprotection Studies: Insights From Randomized Controlled Trials. JACC Cardiovasc Imaging 2017;10:230-40.

11. Kowallick JT, Morton G, Lamata P, Jogiya R, Kutty S, Lotz J, Hasenfuß G, Nagel E, Chiribiri A, Schuster A. Inter-study reproducibility of left ventricular torsion and torsion rate quantification using $M R$ myocardial feature tracking. J Magn Reson Imaging 2016;43:128-37.

12. Taylor RJ, Moody WE, Umar F, Edwards NC, Taylor TJ, Stegemann B, Townend JN, Hor KN, Steeds RP, Mazur W, Leyva F. Myocardial strain measurement with featuretracking cardiovascular magnetic resonance: normal values. Eur Heart J Cardiovasc Imaging 2015;16:871-81.

13. Qu YY, Paul J, Li H, Ma GS, Buckert D, Rasche V. Left ventricular myocardial strain quantification with two- and three-dimensional cardiovascular magnetic resonance based tissue tracking. Quant Imaging Med Surg 2021;11:1421-36.

14. Almutairi HM, Boubertakh R, Miquel ME, Petersen SE. Myocardial deformation assessment using cardiovascular magnetic resonance-feature tracking technique. $\mathrm{Br} \mathrm{J}$ Radiol 2017;90:20170072.

15. Cho GY, Marwick TH, Kim HS, Kim MK, Hong $\mathrm{KS}$, Oh DJ. Global 2-dimensional strain as a new prognosticator in patients with heart failure. J Am Coll 
Cardiol 2009;54:618-24.

16. Buss SJ, Emami M, Mereles D, Korosoglou G, Kristen AV, Voss A, Schellberg D, Zugck C, Galuschky C, Giannitsis E, Hegenbart U, Ho AD, Katus HA, Schonland SO, Hardt SE. Longitudinal left ventricular function for prediction of survival in systemic light-chain amyloidosis: incremental value compared with clinical and biochemical markers. J Am Coll Cardiol 2012;60:1067-76.

17. Korosoglou G, Gitsioudis G, Voss A, Lehrke S, Riedle N, Buss SJ, Zugck C, Giannitsis E, Osman NF, Katus HA. Strain-encoded cardiac magnetic resonance during high-dose dobutamine stress testing for the estimation of cardiac outcomes: comparison to clinical parameters and conventional wall motion readings. J Am Coll Cardiol 2011;58:1140-9.

18. Romano S, Judd RM, Kim RJ, Kim HW, Klem I, Heitner J, Shah DJ, Jue J, White BE, Shenoy C, Farzaneh-Far A. Association of Feature-Tracking Cardiac Magnetic Resonance Imaging Left Ventricular Global Longitudinal Strain With All-Cause Mortality in Patients With Reduced Left Ventricular Ejection Fraction. Circulation 2017;135:2313-5.

19. Romano S, Judd RM, Kim RJ, Kim HW, Klem I, Heitner JF, Shah DJ, Jue J, White BE, Indorkar R, Shenoy C, Farzaneh-Far A. Feature-Tracking Global Longitudinal Strain Predicts Death in a Multicenter Population of Patients With Ischemic and Nonischemic Dilated Cardiomyopathy Incremental to Ejection Fraction and Late Gadolinium Enhancement. JACC Cardiovasc Imaging 2018;11:1419-29.

20. Buss SJ, Breuninger K, Lehrke S, Voss A, Galuschky C, Lossnitzer D, Andre F, Ehlermann P, Franke J, Taeger T, Frankenstein L, Steen H, Meder B, Giannitsis E, Katus HA, Korosoglou G. Assessment of myocardial deformation with cardiac magnetic resonance strain imaging improves risk stratification in patients with dilated cardiomyopathy. Eur Heart J Cardiovasc Imaging 2015;16:307-15.

21. Romano S, Judd RM, Kim RJ, Heitner JF, Shah DJ, Shenoy C, Evans K, Romer B, Salazar P, FarzanehFar A. Feature-Tracking Global Longitudinal Strain Predicts Mortality in Patients With Preserved Ejection Fraction: A Multicenter Study. JACC Cardiovasc Imaging 2020;13:940-7.

22. Zhang Z, Ma Q, Cao L, Zhao Z, Zhao J, Lu Q, Zeng L, Zhang M, Pohost GM, Li K. Correlation between left ventricular myocardial strain and left ventricular geometry in healthy adults: a cardiovascular magnetic resonance-feature tracking study. Int J Cardiovasc Imaging
2019;35:2057-65.

23. Mordi I, Bezerra H, Carrick D, Tzemos N. The Combined Incremental Prognostic Value of LVEF, Late Gadolinium Enhancement, and Global Circumferential Strain Assessed by CMR. JACC Cardiovasc Imaging 2015;8:540-9.

24. Mangion K, Carrick D, Carberry J, Mahrous A, McComb C, Oldroyd KG, Eteiba H, Lindsay M, McEntegart M, Hood S, Petrie MC, Watkins S, Davie A, Zhong X, Epstein FH, Haig CE, Berry C. Circumferential Strain Predicts Major Adverse Cardiovascular Events Following an Acute ST-Segment-Elevation Myocardial Infarction. Radiology 2019;290:329-37.

25. Eitel I, Stiermaier T, Lange T, Rommel KP, Koschalka A, Kowallick JT, Lotz J, Kutty S, Gutberlet M, Hasenfuß G, Thiele H, Schuster A. Cardiac Magnetic Resonance Myocardial Feature Tracking for Optimized Prediction of Cardiovascular Events Following Myocardial Infarction. JACC Cardiovasc Imaging 2018;11:1433-44.

26. Gavara J, Rodriguez-Palomares JF, Valente F, Monmeneu JV, Lopez-Lereu MP, Bonanad C, et al. Prognostic Value of Strain by Tissue Tracking Cardiac Magnetic Resonance After ST-Segment Elevation Myocardial Infarction. JACC Cardiovasc Imaging 2018;11:1448-57.

27. Yoon YE, Kang SH, Choi HM, Jeong S, Sung JM, Lee SE, Cho I, Cho GY, Chang HJ, Chun EJ. Prediction of infarct size and adverse cardiac outcomes by tissue tracking-cardiac magnetic resonance imaging in STsegment elevation myocardial infarction. Eur Radiol 2018;28:3454-63.

28. Korosoglou G, Giusca S, Montenbruck M, Patel AR, Lapinskas T, Götze C, Zieschang V, Al-Tabatabaee S, Pieske B, Florian A, Erley J, Katus HA, Kelle S, Steen H. Fast Strain-Encoded Cardiac Magnetic Resonance for Diagnostic Classification and Risk Stratification of Heart Failure Patients. JACC Cardiovasc Imaging 2021;14:1177-88.

29. Buckert D, Kelle S, Buss S, Korosoglou G, Gebker R, Birkemeyer R, Rottbauer W, Katus H, Pieske B, Bernhardt P. Left ventricular ejection fraction and presence of myocardial necrosis assessed by cardiac magnetic resonance imaging correctly risk stratify patients with stable coronary artery disease: a multi-center all-comers trial. Clin Res Cardiol 2017;106:219-29.

30. Coleman GC, Shaw PW, Balfour PC Jr, Gonzalez JA, Kramer CM, Patel AR, Salerno M. Prognostic Value of Myocardial Scarring on CMR in Patients With Cardiac Sarcoidosis. JACC Cardiovasc Imaging 2017;10:411-20. 
31. Hachamovitch R, Hayes SW, Friedman JD, Cohen I, Berman DS. Comparison of the short-term survival benefit associated with revascularization compared with medical therapy in patients with no prior coronary artery disease undergoing stress myocardial perfusion single photon emission computed tomography. Circulation 2003;107:2900-7.

32. Schumacher SP, Everaars H, Stuijfzand WJ, Huynh JW, van Diemen PA, Bom MJ, de Winter RW, van Loon RB, van de Ven PM, van Rossum AC, Opolski MP, Nap A, Knaapen P. Coronary collaterals and myocardial viability in patients with chronic total occlusions. EuroIntervention 2020;16:e453-61.

33. Li JN, He Y, Dong W, Zhang LJ, Mi HZ, Zhang DF, Huang RC, Song XT. Comparison of cardiac MRI with PET for assessment of myocardial viability in patients with coronary chronic total occlusion. Clin Radiol 2019;74:410. e1-9.

Cite this article as: Zhang $\mathrm{L}$, Tian J, Yang X, Liu J, He Y, Song X. Quantification of strain analysis and late gadolinium enhancement in coronary chronic total occlusion: a cardiovascular magnetic resonance imaging follow-up study. Quant Imaging Med Surg 2022;12(2):1484-1498. doi: 10.21037/qims-21-702 
Supplementary

Table S1 Medical history for the CTOs with left ventricular ejection fraction decreased or preserved

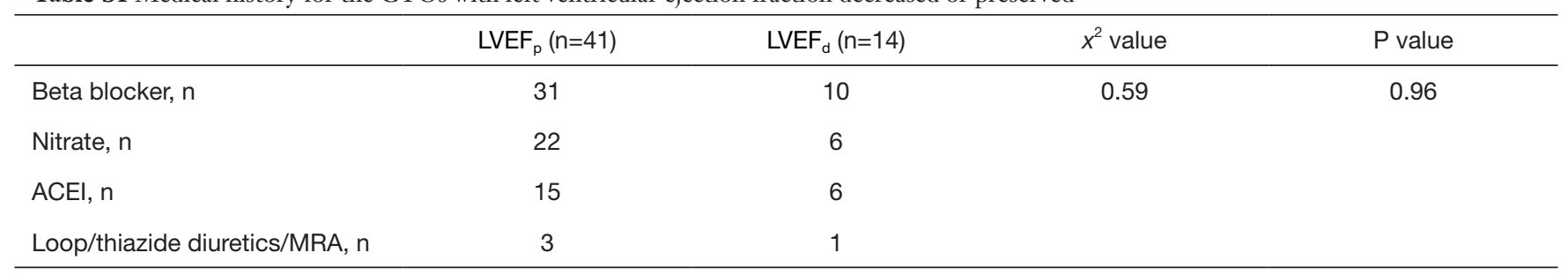

$\mathrm{LVEF}_{\mathrm{d}}$, left ventricular ejection fraction decreased; $\mathrm{LVEF}_{\mathrm{p}}$, left ventricular ejection fraction preserved. 